\title{
UNA ESPECIE NUEVA DE PACHYPHYTUM (CRASSULACEAE) PARA LA FLORA DE JALISCO, MÉXICO
}

\section{Emmanuel Pérez-Calix ${ }^{1}$, Ignacio García Ruiz ${ }^{2}$ y Miguel Cházaro Basáñez ${ }^{3}$}

\author{
${ }^{1}$ Instituto de Ecología, A.C., Centro Regional del Bajío, Apdo. postal 386, 61600 \\ Pátzcuaro, Michoacán, México. emmanuel.perezcalix@inecol.edu.mx \\ ${ }_{2}^{2}$ Instituto Politécnico Nacional, Centro Interdisciplinario de Investigación para el \\ Desarrollo Integral Regional, Unidad Michoacán. Justo Sierra 28, 59510 Jiquilpan, \\ Michoacán, México. igarciar@ipn.mx \\ ${ }^{3}$ Departamento de Geografía, Universidad de Guadalajara, Avenida Maestros y M. \\ Bárcena, 44260 Guadalajara, Jalisco, México.
}

\section{RESUMEN}

Se describe y propone como especie nueva para la ciencia a Pachyphytum contrerasii (Crassulaceae). El nuevo taxon se ubica en la sección Pachyphytum Moran, por llevar las brácteas imbricadas en el cincino joven, tener la corola más corta que el cáliz y por la presencia de una mancha roja evidente en la cara interna de los segmentos de la corola. La especie más semejante morfológicamente es $P$. machucae I. García, Glass et Cházaro, de la cual $P$. contrerasii difiere en diversos caracteres de la flor, tales como: el largo del pedicelo, la relación entre el largo de la corola y el del cáliz y la coloración de la cara interna de los pétalos, estambres y pistilo.

Palabras clave: clado Acre, Echeverioideae, Pachyphytum machucae, taxonomía.

\begin{abstract}
Pachyphytum contrerasii (Crassulaceae) is proposed and described here as a new species from central Jalisco, western Mexico. This taxon belongs to the section Pachyphytum Moran, on account of its imbricate bracts of the young cincinnus, of its corolla shorter than the calyx and of its the conspicuous red dot on the inner face of the corolla segments. In terms of morphology and geography the closest relative is Pachyphytum machucae I. Garcia, Glass \& Cházaro, from northwestern Michoacan, In addition to its geographical distribution, Pachyphytum contrerasii is separated from this species by the possession of pedicels 2-4 mm long, a corolla $3 \mathrm{~mm}$ long that is shorter than the pair of larger sepals, and bicolored inner face of the petals, stamens and pistil. Besides being a new species this is the first report of the genus Pachyphytum for Jalisco state.
\end{abstract}

Key words: clado Acre, Echeverioideae, Pachyphytum machucae, taxonomy. 
El género Pachyphytum (Crassulaceae), en su ubicación tradicional, pertenece a la subfamilia Echeverioideae (sensu Berger, 1930), mientras que las propuestas modernas, como la de Hart (1995) lo agrupan con Echeveria DC., Graptopetalum Rose, Lenophyllum Rose, Tacitus Moran, Thompsonella Rose y Villadia Rose en el clado Acre (subfamilia Sedoideae; tribu Sedeae; subtribu Sedinae). Se diferencia de los demás por presentar un par de apéndices escamosos en la cara interna de cada pétalo. Como se conoce en la actualidad, el género consta de 17 especies de distribución restringida al centro de México, desde la región sureste de Tamaulipas al centro y noroeste de Michoacán, abarcando porciones de los estados de Hidalgo, San Luis Potosí, Querétaro, Guanajuato y Aguascalientes. La mayoría de las especies presentan distribución muy restringida, pues con excepción de Pachyphytum compactum Rose, P. hookeri (Salm-Dyck) A. Berger, P. glutinicaule Moran y P. viride E. Walther, las demás se registran únicamente de la localidad en que se colectó el tipo y de áreas cercanas. Cabe mencionar que la especie que aquí se describe es la primera del género que se localiza en el estado de Jalisco, lo que a su vez amplía la distribución conocida para el grupo hacia el occidente de México.

Durante una exploración realizada en busca de orquídeas en la cascada Cola de Caballo, en el municipio de Zapopan, Jalisco, el señor Ignacio Contreras Villaseñor halló creciendo sobre las rocas una planta suculenta que llamó su atención y colectó algunos ejemplares que fueron determinados como pertenecientes al género Pachyphytum. Sin embargo, se encontró que no corresponde a ninguna de las especies descritas con anticipación y debido a ello se propone como nueva para la ciencia y se le dedica el nombre a su descubridor.

Pachyphytum contrerasii E. Pérez-Calix, I. García et Cházaro, sp. nov. TIPO: México. Jalisco: municipio de Zapopan, cantil rocoso contiguo a la Cascada "Cola de Caballo" junto al Mirador "Dr. Atl”, alt. 1450 m. 24 sep. 2001. M. Cházaro B., L. F. Gómez y J. R. Gómez 8157 (holotipo: IEB; isotipos: CIMI, ENCB, MEXU y XAL). (Fig. 1.)

Planta suffrutescens, succulenta, glabra, pendula vel decumbens, caules simplices vel ramosi, usque $20 \mathrm{~cm}$ longi; folia rosulam usque ad $12 \mathrm{~cm}$ diametro formantia, laminae ambitu anguste ellipticae usque lanceolatae, apice obtusae, 4-7.5 cm longae, $1.5-2.5 \mathrm{~cm}$ latae ad partem mediam, 5-7 mm crassae, viridi-glaucae usque roseae ad basem, griseo-lavandulaceae usque viridi-caerulescentes ad partem mediam et apicem; inflorescentia cincinniformis, pedunculus usque $25 \mathrm{~cm}$ longus, 12-24 floribus, bracteae imbricatae in cincinno juveni, oblongo-ovatae, ad apicem 


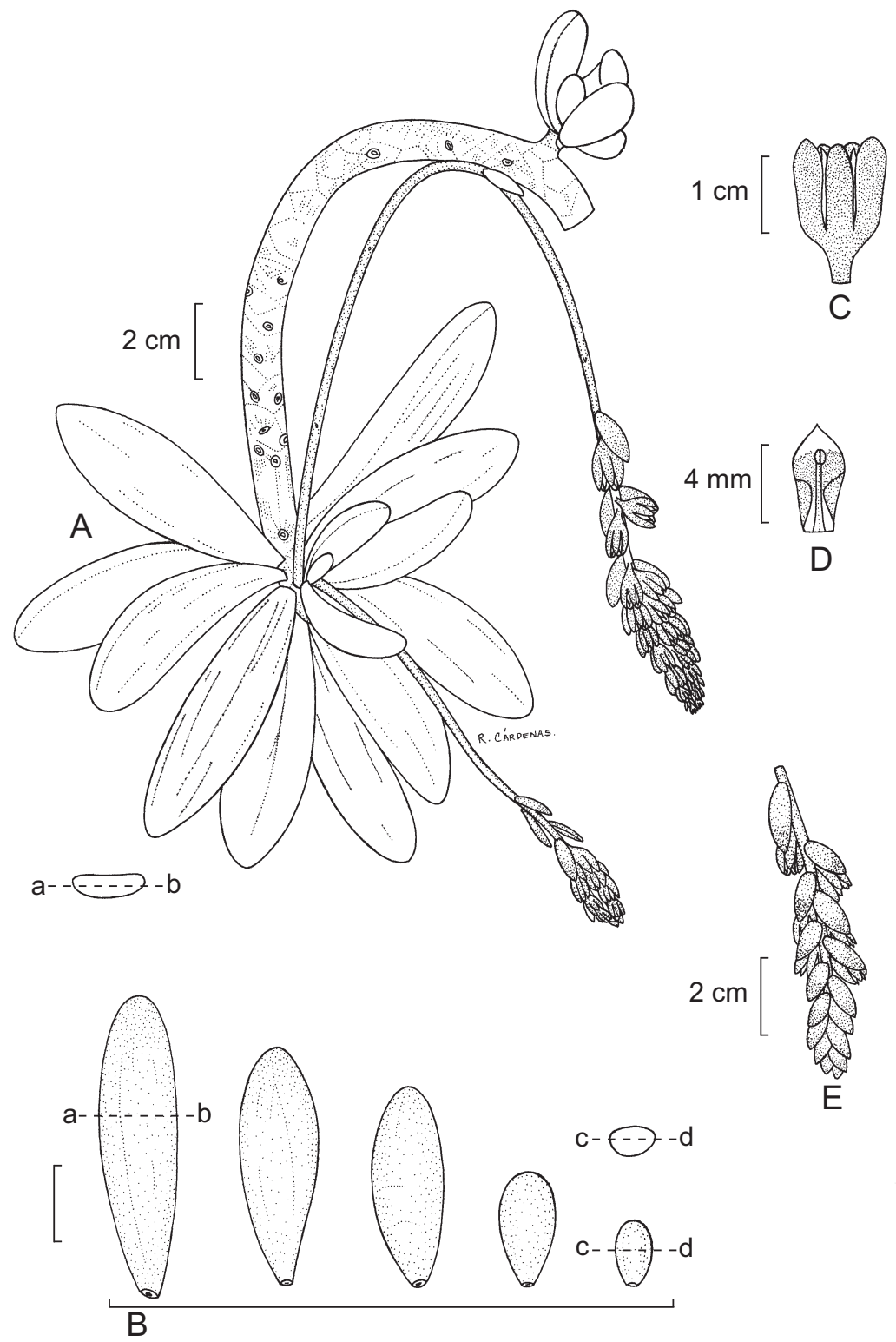

Fig. 1. Pachyphytum contrerasii E. Pérez-Calix, I. García et Cházaro. A. hábito; B. variación de las hojas: a-b y c-d, secciones transversales de las láminas foliares; C. flor; D. cara interna de un segmento de la corola; E. inflorescencia en vista dorsal, mostrando las brácteas imbricadas. (Ilustración de R. Cárdenas Soriano, del ejemplar I. García y J. A. Machuca $5569)$. 
obtusatae et mucronulatae, 6-16 mm longae, 3-9 mm latae, 2-5 $\mathrm{mm}$ crassae, virides vel olivaceae, glaucae; calyx corollam superans, segmentis oblongis inaequalibus; corolla subcampanulata, ad basem connata, lobi oblongi, 7-8 mm longi, 3-3.5 mm lati, ad apicem acuti, extus dilute virides, ad apicem fusci, intus dilute virides, maculam scarlatinam, $2.5 \mathrm{~mm}$ diametro ferentes, segmentarum appendices ovali-subcirculares, $1 \mathrm{~mm}$ longi, scarlatini.

Planta sufrutescente, suculenta, glabra, glauca, colgante a decumbente; tallos simples o ramificados cerca de la base, hasta de $20 \mathrm{~cm}$ de largo y de $10-15 \mathrm{~mm}$ de diámetro cerca de la base; hojas formado una roseta hasta de $12 \mathrm{~cm}$ de diámetro, laxa en el ápice del tallo; láminas estrechamente elípticas a oblanceoladas en contorno, estrechamente elípticas en sección transversal, ápice romo, de 4-7.5 cm de largo, de 1.5-2.5 $\mathrm{cm}$ de ancho en la parte media, de 5-7 $\mathrm{mm}$ de grueso, glaucas, de color verde glauco a rosado en la base, gris-lavanda a verde-azulado en la parte media y en el ápice; inflorescencia en forma de cincino, pedúnculo hasta de $25 \mathrm{~cm}$ de largo y de 3-5 mm de diámetro cerca de la base, de color rosado, cincino de 10-16 cm de largo, con 12-24 flores; brácteas imbricadas en el cincino joven, alternas, oblongoovadas, ápice obtuso, mucronulado, ligeramente sagitadas en la base, de 6-16 mm de largo, de 3-9 mm de ancho, de 2-5 $\mathrm{mm}$ de grueso, glaucas, de color verde a verde olivo, rosadas en la base y con la edad; pedicelos de $2-4 \mathrm{~mm}$ de largo y de $2 \mathrm{~mm}$ de diámetro, glaucos, de color verde; cáliz mayor que la corola, sépalos 5 , fusionados en la base formando un tubo de 3-3.5 $\mathrm{mm}$ de alto y $6.5 \mathrm{~mm}$ de diámetro, segmentos oblongos, desiguales entre sí en largo y ancho, dos mayores de 9-12 mm de largo por 3.5-6 $\mathrm{mm}$ de ancho, de 1.3-1.5 $\mathrm{mm}$ de grueso, tres menores de 6-9 $\mathrm{mm}$ de largo, de 3-4 mm de ancho, de 0.6-0.7 mm de grueso, glaucos, de color verde, ápice obtuso y mucronulado; corola subcampanulada, pétalos 5 , connados cerca de la base, lóbulos oblongos, de 7-8 mm de largo, 3-3.5 mm de ancho, ápice agudo, cara externa de color verde muy claro con el ápice ligeramente más oscuro, cara interna verde clara con una mancha oval de color rojo escarlata de $2.5 \mathrm{~mm}$ de diámetro, apéndices de los segmentos oval-subcirculares, de $1 \mathrm{~mm}$ de largo, de color rojo escarlata; filamentos antisépalos de $4.5 \mathrm{~mm}$ de largo, los antipétalos adnados, de 3-4 $\mathrm{mm}$ de largo, anteras de $0.5 \mathrm{~mm}$ de largo; nectario de $1 \mathrm{~mm}$ de largo, de color amarillo; ovario de 3 $\mathrm{mm}$ de alto por $3 \mathrm{~mm}$ de diámetro, estilos de $1 \mathrm{~mm}$ de largo, de color rojo, estigmas globosos, de $0.2 \mathrm{~mm}$ de diámetro; folículos de $5 \mathrm{~mm}$ de largo; semillas numerosas, de $0.2 \mathrm{~mm}$ de largo, de color café.

Pachyphytum contrerasii lleva las brácteas imbricadas en el cincino joven, la corola más corta que el cáliz y tiene una mancha roja en la cara interna de los pétalos, características que lo ubican en la sección Pachyphytum (sensu Moran, 1968). Mor- 
fológicamente, la especie nueva tiene mayor semejanza con P. machucae I. García, Glass et Cházaro (García et al., 1999), del que se diferencia de la siguiente manera: $P$. contrerasii tiene pedicelos de $2-4 \mathrm{~mm}$ de largo y de $2 \mathrm{~mm}$ de diámetro, mientras que los de $P$. machucae miden de 5-10 $\mathrm{mm}$ de largo y de 1.5-2 $\mathrm{mm}$ de diámetro. Además, la corola de la nueva especie es $3 \mathrm{~mm}$ más corta que los sépalos mayores, mientras que la de $P$. machucae es tan larga o brevemente más corta que los sépalos mayores; y, por último, en $P$. contrerasii la cara interna de los pétalos, el pistilo y los estambres son bicolores, de color amarillo-crema o blanquecino en las tres cuartas partes inferiores y de color escarlata hacia el ápice, a su vez, en $P$. machucae estas partes de la flor son totalmente de color de rosa.

Paratipos: MÉXICO. Jalisco: municipio Zapopan, Salto cascada "Cola de Caballo", mirador Dr. Atl, carretera Guadalajara-Saltillo aprox. km 14; I. García \& J. A. Machuca 5569 (CIMI). Misma localidad, M. Cházaro, M. Mendoza y C. Glass 7790 (IEB).

Fenología: la nueva especie se ha observado en floración de noviembre a marzo, cada planta produce hasta tres inflorescencias por año.

Distribución geográfica y ecológica: Pachyphytum contrerasii se conoce únicamente de la localidad en que se colectó el tipo, en el centro-norte del estado de Jalisco. Habita en medio del bosque tropical caducifolio, sobre taludes verticales de rocas ígneas, en laderas con orientación norte, en altitudes de 1380 a 1450 m, en donde crece junto con Tillandsia capitata Griseb.

\section{AGRADECIMIENTOS}

Estamos muy agradecidos con las siguientes personas por su ayuda en el trabajo de campo, sin la cual hubiera sido imposible conseguir especímenes de la planta: Ignacio Contreras, Oscar M. Valencia, J. A. Machuca, J. Cortés, S. Cortés, A. Galván, A. Larios; en especial con J. Rodrigo y L. Felipe Gómez Jolly, quienes descendieron por el cantil para obtener las muestras. También damos las gracias al Dr. Jerzy Rzedowski R. y al Dr. Victor Steinmann por la lectura crítica y sugerencias al manuscrito. La ilustración se debe a la inspiración del Sr. Rogelio Cárdenas. El primer autor reconoce el apoyo económico del Instituto de Ecología, A.C. (cuenta 20006), del Consejo Nacional de Ciencia y Tecnología y de la Comisión Nacional 
para el Conocimiento y Uso de la Biodiversidad; el segundo autor agradece a la Comisión de Operación y Fomento para las Actividades Académicas y de Estímulos al Desempeño de los Investigadores del Instituto Politécnico Nacional.

\section{LITERATURA CITADA}

Berger, A. 1930. Crassulaceae. In: Engler, A. y K. Prantl (eds.). Die natürlichen Pflanzenfamilien, ed. 2, 18a. Verlag Wilhelm Engelmann. Leipzig. pp. 352-458.

García, I., C. Glass y M. Cházaro. 1999. Pachyphytum machucae (Crassulaceae) una nueva especie de Michoacán, México. Acta Bot. Mex. 47: 9-14.

Hart, H. 1995. Infrafamilial and generic classification of the Crassulaceae. In: Hart, H. y U. Eggli (eds.). Evolution and systematic of the Crassulaceae. Backhuys Publishers. Leiden. pp. 159-171.

Moran, R. 1968. New subgeneric groups in Echeveria and Pachyphytum. Cact. Suc. (U.S.): 40: 36-42. 